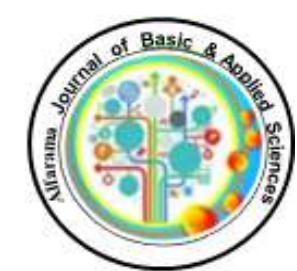

ISSN 2682-275X
Alfarama Journal of Basic \& Applied Sciences

Faculty of Science Port Said University https://ajbas.journals.ekb.eg ajbas@sci.psu.edu.eg

http://sci.psu.edu.eg/en/

January 2022, Volume 3 Issue I

DOI: $10.21608 / A J B A S .2021 .92698 .1064$

Submitted: 25-08-2021

Accepted: 18-10-2021

Pages: 74-87

\title{
Effects of Tripterygium wilfordii extracts on the hematological parameters of solid Ehrlich carcinoma bearing mice
}

\author{
Basma A.Rageh ${ }^{1}$, Mohamed M. Tawfik ${ }^{1}$, Osama A. Abbas ${ }^{1}$, and Ahmad K. Hassan ${ }^{1, *}$ \\ ${ }^{1}$ Zoology Department, Faculty of Science, Port Said University. \\ Corresponding author: Ahkhalaf71@yahoo.com
}

\begin{abstract}
The complete blood picture or count (CBC) and different hematological measurments are widely used in the assessment of many health problems such as cancer prognosis and progression. The roots of Chinese herb Tripterygium wilfordii (TW) is well-defined in Chinese alternative medicine since it is rich in a lot of beneficial components such as terpenoids, alkaloids and steroids. The intratumoral treatment with TW ethanolic and aqueous extracts individually and mix of them at a dose $(16 \mathrm{mg} / \mathrm{kg}$ ) in Ehrlich ascites carcinoma (EAC) bearing mice had ameliorated the different hematological parameters such as red blood cell count (RBCs), hemoglobin concentration, white blood cells (WBCs) count and differential, as well as platelet count near normal values.

Treatment with cisplatin $(2 \mathrm{mg} / \mathrm{kg})$ revealed many undesired hematological side effects like anemia, leukopenia and neutrophilia. Moreover, recently used inflamatory markers as prospective diagnostic parameters for cancer prognosis like monocyte lyphocyte ratio (MLR), neutrophil lymphocyte ratio (NLR), and platelet lymphocyte ratio (PLR) were decreased significantly after treatment with TW extracts. In conclusion, treatment of EAC bearing mice with different extracts of TW could have anticancer activity regarding their remarked effect in improvement of distorted hematological parameters of the EAC bearing mice.
\end{abstract}

\section{Keywords}

Hematological parameters, EAC, Mice, Chinese herb Tripterygium wilfordii.

\section{INTRODUCTION}

Cancer is one of the leading causes of death in the worldwide. Carcinoma of the liver, breast and urinary bladder are the most common malignancies among Egyptians of both sexes. The incidence rate of cancer cases in Egypt was 115,000 in 2013 in both sexes and it is estimated to have increased three-folds by the year 2050. Medical surgery, chemotherapy, and radiotherapy are the most common protocols in cancer treatments [2]. However, most of these regimens could have a number of severe toxicities, like hepatic, gastrointestinal, cardiac, and hematological in particular. Changes in the hematological measurements are 
influenced by cancer, which affects the haemopoetic physiology at different levels [3]. As a result, hematological parameters have become widely used in assessing severity and optimizing treatment for various types of cancer. Furthermore, in cancer patients receiving radiotherapy and/or chemotherapy, hematological severe illnesses such as anemia, leukopenia, increased platelet count, and a decrease in RBC count and hemoglobin level are common [4]. Therefore, complete blood count is routinely required for all cancer patients to the monitor prognosis of several cancers.

Different established hematological markers like erythrocyte indices, white blood cells count, and mean platelet volume or counts are widely used for the prognosis and disease staging of several types of malignancies. Moreover, systemic inflammatory markers such as neutrophil-lymphocyte ratio (NLR), monocyte-lymphocyte ratio (MLR), and plateletlymphocyte ratio (PLR) have been recently used as simple, inexpensive, and effective prognostic tumor markers in cancer with less or no patient discomfort. Several studies have elucidated that high NLR, MLR, and PLR values are associated with a prognosis in patients with esophageal, colorectal, lung, breast cancer, and malignant melanoma [5].

More than half of the currently used anticancer drugs are derived from natural products. Meanwhile, they are a rich source of potent but less toxic anticancer agents than chemotherapeutics. The Chinese herb Tripterygium wilfordii is (TW) a well-known Chinese traditional medicine. The leaves and flowers of this plant are highly poisonous, but the root is a source of various therapeutic components. Several biologically active terpenoids, alkaloids and steroids have been identified from this herb root. TW exhibits pharmacological activities against inflammation [6], autoimmune disorders [7], fibrosis, atherosclerosis, neurodegeneration [8], kidney diseases [9] and cancer [10]. The aim of this study was to investigate the effect of different extracts of TW on the haematological parameters in the EAC solid tumor model along with the effect of cisplatin, as a standard widely used chemotherapeutic medication.

\section{MATERIALS AND METHODS}

\section{Collection and preparation of the plant material}

The roots of Tripterygium wilfordii (TW) were bought from Taobao, China and identified with the help of a taxonomist in the Botany Department, Faculty of Science, Port Said University. The roots were collected with acceptable bio-conservative methods and were properly sorted out, cleaned, and transported to the Biology laboratories at Port Said University, for drying and crushing. The roots are chopped into small pieces and grounded into a fine homogeneous powder using an electric mill followed by sieving through a mesh sieve and stored at room temperature until the extraction.

\section{Plant Extraction}

For ethanol extraction, the powder was rinsed three times in $95 \%$ ethanol for 2 hours with occasional shaking and filtered through four sheets of gauze, then twice on filter paper and dried by rotatory evaporation under reduced pressure to yield the ethanol extract. The crude extract was then kept at $-20{ }^{\circ} \mathrm{C}$ in sterile universal bottles until used [11]. For aqueous extract, the powder was extracted with distilled water as described in the Encyclopedia of Chinese Medicines with modifications. The extract was filtered by using filter paper and the filtrate was collected and frozen in an ice cube container. The frozen ice cube was freeze- 
dried (i.e. lyophilisation) to obtain a concentrated aqueous extract in powder form. The crude extract was then kept at $-20{ }^{\circ} \mathrm{C}$ in sterile universal bottles [12] .

\section{Experimental animals}

A total of 60 female Swiss albino mice, weighing 22-25 g, were purchased from the National Cancer Institute, Cairo, Egypt. Animals were separated into six groups, each of ten animals, and housed at the Animal House, Faculty of Science, Port Said University in plastic cages in a well-ventilated room $\left(26 \pm 2{ }^{\circ} \mathrm{C}\right)$ with a relative humidity of $(40 \pm 2 \%)$, a $12 \mathrm{hr}$ light/12 hr dark cycle and free access to feed and water. All animal procedures and care were performed using guidelines from the international guiding principles for animal research.

\section{Induction of Ehrlich Solid Tumor}

A line of Ehrlich ascites carcinoma cells (EAC) used in the present study has been kindly supplied by the National Cancer Institute (NCI), Cairo University, Egypt. It was maintained in female Swiss albino mice by weekly intraperitoneal injection of $2.5 \times 10^{6}$ tumor cells per mouse (in the right thigh of the lower limb of each mouse). The solid form was obtained by the intramuscular inoculation of $0.2 \mathrm{ml}$ of EAC, which contained $2.5 \times 10^{6}$ viable EAC cells. Mice with a palpable solid tumor that developed within 10 days after inoculation were used in the study [13].

\section{Experimental Design}

Group I (Negative control group): 10 normal mice received $0.2 \mathrm{ml}$ of saline daily for 14 consecutive days. Group II (Positive control group; $n=10$ ) mice were inoculated with EAC cells and received $0.2 \mathrm{ml}$ of saline intratumorally daily for 14 consecutive days. Group III (ethanolic extract treated group); 10 mice were inoculated with EAC cells and received (1/10 LD50) $16 \mathrm{mg} / \mathrm{kg}$ TW extract intratumorally for 14 consecutive days [14]. Group IV (aqueous extract treated group): 10 mice were inoculated with EAC cells and received (1/10 LD50) $16 \mathrm{mg} / \mathrm{kg}$ TW daily for 14 consecutive days [14]. Group V (Mix extract treated group): 10 mice were inoculated with EAC cells and received $(8 \mathrm{mg} / \mathrm{kg}$ ethanolic extract +8 $\mathrm{mg} / \mathrm{kg}$ aqueous extract) intratumorally daily for 14 consecutive days. Group VI (Cisplatin treated group): 10 normal mice were inoculated with EAC cells and received $2 \mathrm{mg} / \mathrm{kg}$ of cisplatin intratumorally for 14 consecutive days [15].

\section{Determination of hematological parameters}

Hematological parameters and indices were determined from unclotted blood samples using standard protocols as described by [16]. The erythrocyte number, hemoglobin concentration (HB), mean corpuscular volume (MCV), mean corpuscular hemoglobin $(\mathrm{MCH})$, mean corpuscular hemoglobin concentration (MCHC), red cell distribution width (RDW) and platelets were determined. Also, white blood cell, neutrophils, monocytes, lymphocytes, eosinophils, and basophil counts were evaluated by a Sysmex XN-550 automated hematology analyzer (Germany). The resulted data was confirmed by a hemocytometer using a bright microscope (ZEISS Primo Star). 


\section{Statistical analysis}

The data was subjected to statistical analysis with the level of significance set at $\mathrm{P} \leq 0.05$ ) using the statistical package for social sciences (SPSS) version 20 to determine any significant relationship between the groups. Results were expressed as mean \pm standard error of mean (S.E.M).

\section{RESULTS}

\section{Effects of TW extracts on erythrocytes and related parameters}

All root extracts of TW induced changes in erythrocytes and related parameters. Treatment with ethanolic, aqueous and a mixture of TW significantly increased the level of $\mathrm{Hb}$ and RBC count when compared with the EAC positive control and cisplatin treated groups $(\mathrm{P} \leq 0.05)$. Interestingly, these latter parameters were non-significant $(\mathrm{P} \geq 0.05)$ as compared to control healthy subjects (Figs $1 \& 2$ ). There was a significant decrease $(P \leq 0.05)$ in the RDW of the TW treated groups when compared with the EAC and cisplatin-treated groups (Table 1).

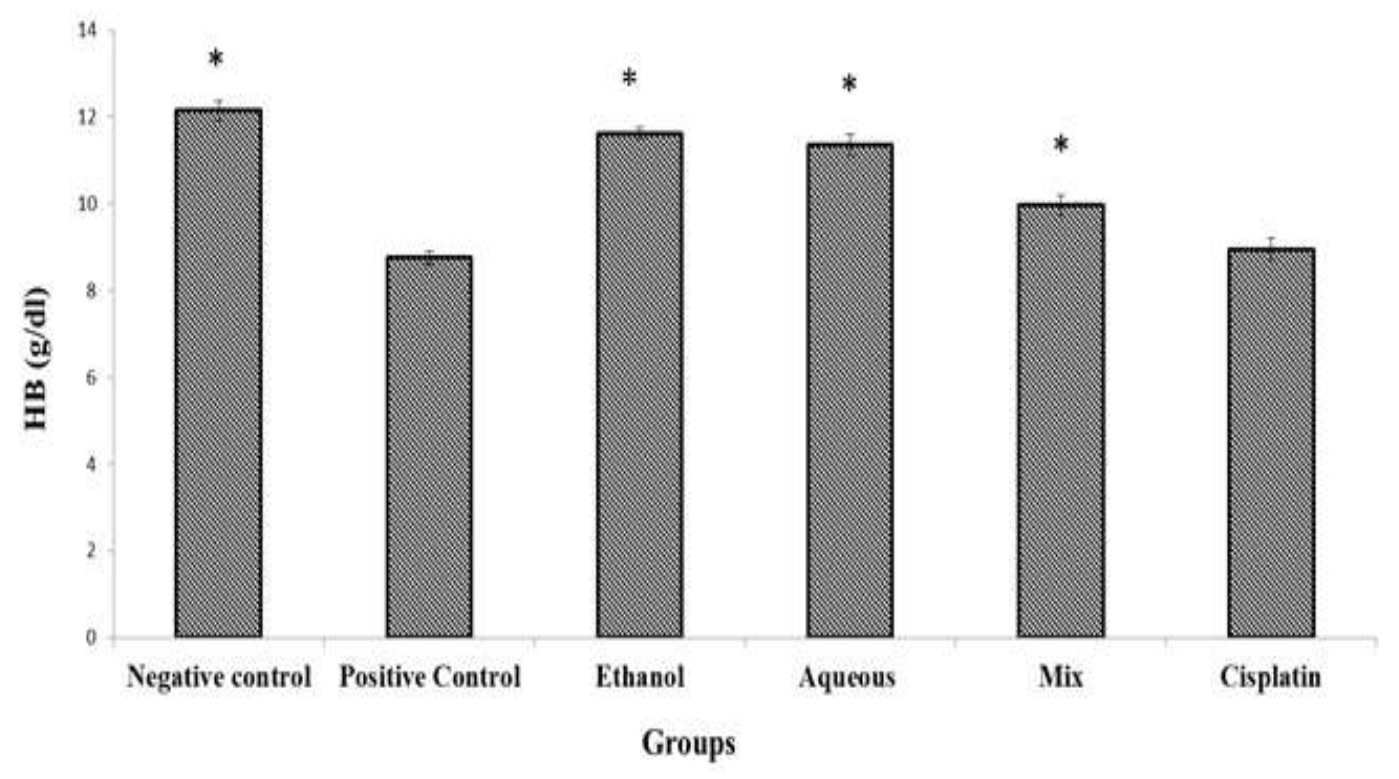

Figure 1. Changes in HB level in the blood of EAC-bearing mice after 14 days of treatment with TW extracts and cisplatin. The results are expressed as means \pm S.E.M. $(n=6)$. Statistically significant differences between different groups compared with EAC positive control group are denoted by $*(\mathrm{P} \leq 0.05)$. 


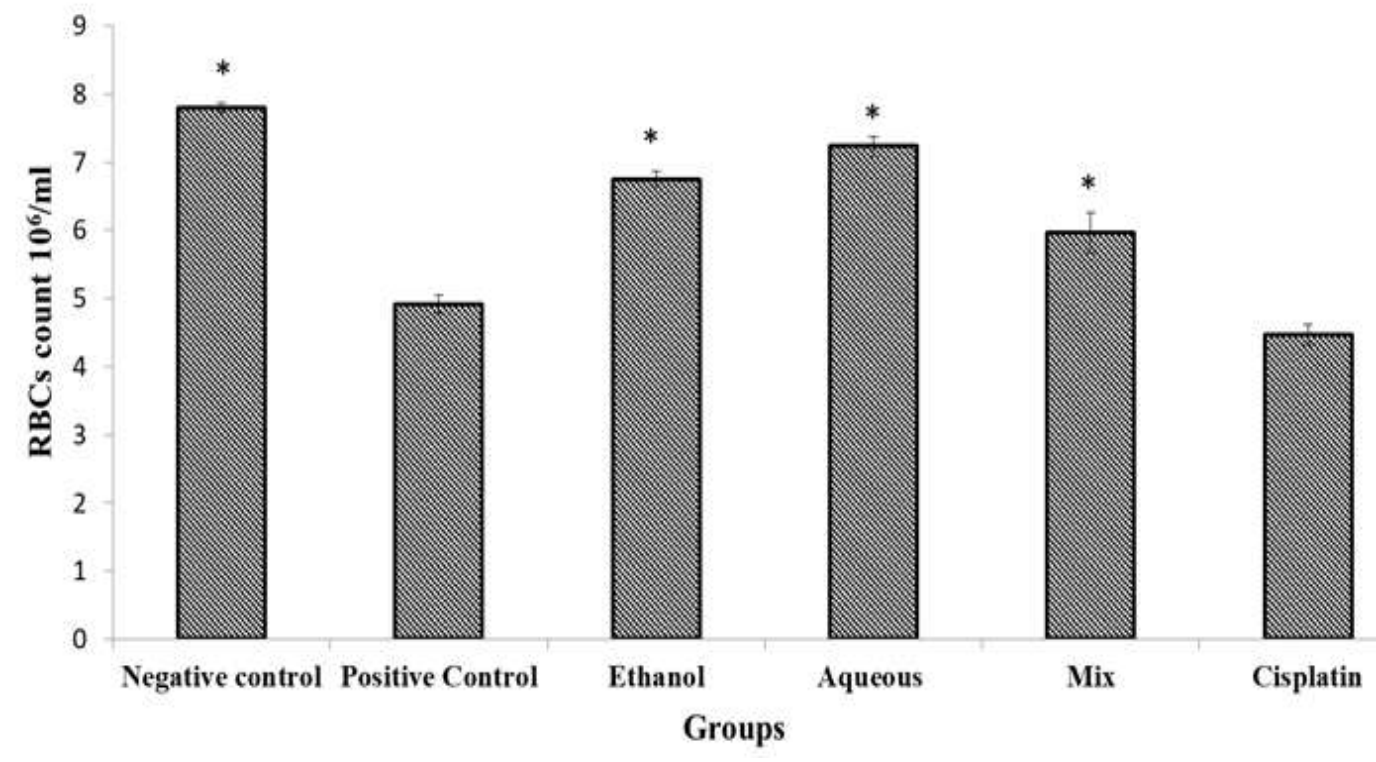

Figure 2. Changes in RBCs count in the blood of EAC-bearing mice after 14 days of treatment with TW extracts and cisplatin.

Table 1 Effects of TW extracts and cisplatin treatment on erythrocytic hematological parameters of different mice groups.

\begin{tabular}{|c|c|c|c|c|c|c|}
\hline \multirow{2}{*}{ Parameter } & \multicolumn{7}{|c|}{ Groups } \\
\cline { 2 - 7 } & Negative & EAC & Ethanol & Aqueous & Mix & Cisplatin \\
\hline MCV & $46 \pm 0.08$ & $43.7 \pm 0.3$ & $45.1 \pm 0.6$ & $44.7 \pm 0.7$ & $45.8 \pm 0.3$ & $46.2 \pm 0.3$ \\
\hline MCH & $14.1 \pm 0.02$ & $13.6 \pm 0.12$ & $14.25 \pm 0.18$ & $14.4 \pm 0.2$ & $14.3 \pm 0.3$ & $14.3 \pm 0.14$ \\
\hline MCHC & $32.7 \pm 0.4$ & $30.6 \pm 0.46$ & $31.65 \pm 0.46$ & $32.47 \pm 0.9$ & $28.7 \pm 0.96$ & $33.38 \pm 0.5$ \\
\hline RDW & $29.2 \pm 0.5^{*}$ & $33.7 \pm 0.9$ & $30.03 \pm 0.51^{*}$ & $30.2 \pm 0.6^{*}$ & $30.4 \pm 0.7 *$ & $33.6 \pm 0.7$ \\
\hline Hematocrit & $35.7 \pm 0.7$ & $26.0 \pm 0.99$ & $34.13 \pm 0.45$ & $34.1 \pm 0.76$ & $33.8 \pm 0.9$ & $26.37 \pm 0.8$ \\
\hline
\end{tabular}

The results are expressed as means \pm S.E.M. $(n=6)$. Statistically significant differences between different groups compared with EAC positive control group are denoted by * $(\mathrm{P}$ $<0.05)$.

\section{Effects of TW extracts on total WBC and differential WBC counts in mice}

The root extracts of TW induced marked changes in total and differential WBC counts in the different mice groups. There was a significant decrease in the levels of total WBC count in the ethanolic, aqueous, mixed and cisplatin treated groups $(\mathrm{P} \leq 0.01)$ when compared with the 
EAC positive control group (Fig 3). The TW extract caused a significant reduction $(\mathrm{P} \leq 0.05)$ in neutrophil percentage when compared to the EAC positive control group. Contrarily, a highly significant increase $(\mathrm{P} \leq 0.05)$ in the percentage of lymphocytes was recorded in the TW treated groups (Fig 4).

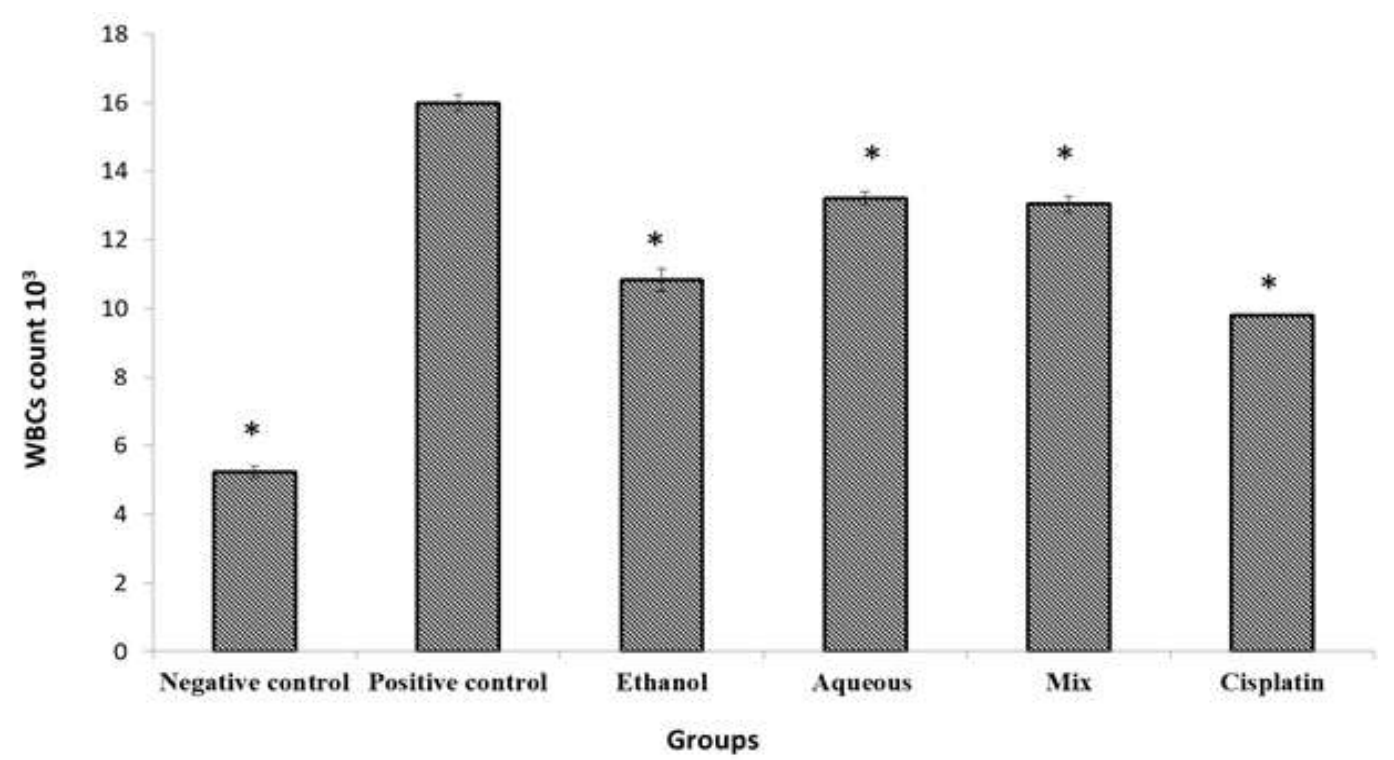

Figure 3. Effect of TW extracts and cisplatin on WBCs count of EAC-bearing mice after 14 days of treatment. The results are expressed as Mean \pm S.E.M. $(n=6)$. Statistically significant differences between experimental groups compared with EAC positive control group are denoted by $*(\mathrm{P} \leq 0.05)$. WBCs, white blood cells.

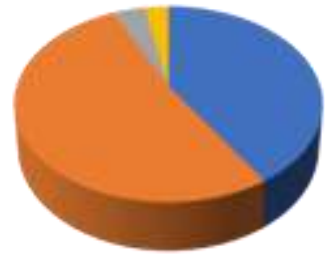

Negative control

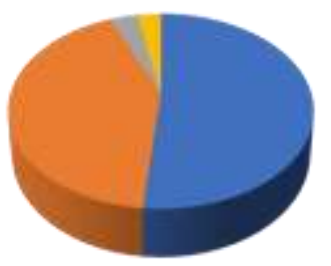

Aqueous

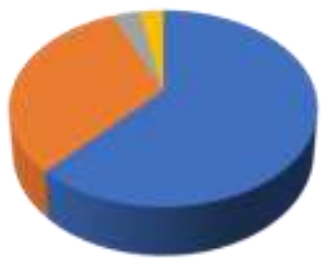

Pesitive control

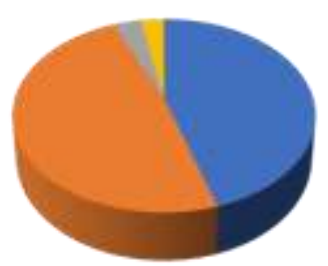

Mis

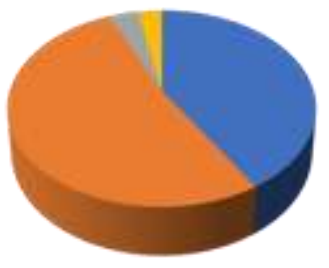

Ethanol

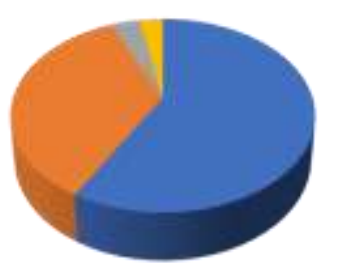

Neutros

wyaphs

$=$ Mosos $\%$

Ensino\%

$n$ Baw \%

Figure 4. Pie chart showing WBCs differentiation in different treated groups after 14 days of treatment with TW extracts and cisplatin. 


\section{Effects of TW extracts on the total platelets count}

Treatment with TW root extracts and cisplatin had significantly decreased platelet count $(\mathrm{P} \leq 0.05)$ in all the treated groups compared with EAC positive control group. Meanwhile, Platelets count was significantly increased in the EAC non treated group compared with the normal negative control group (Fig 5).

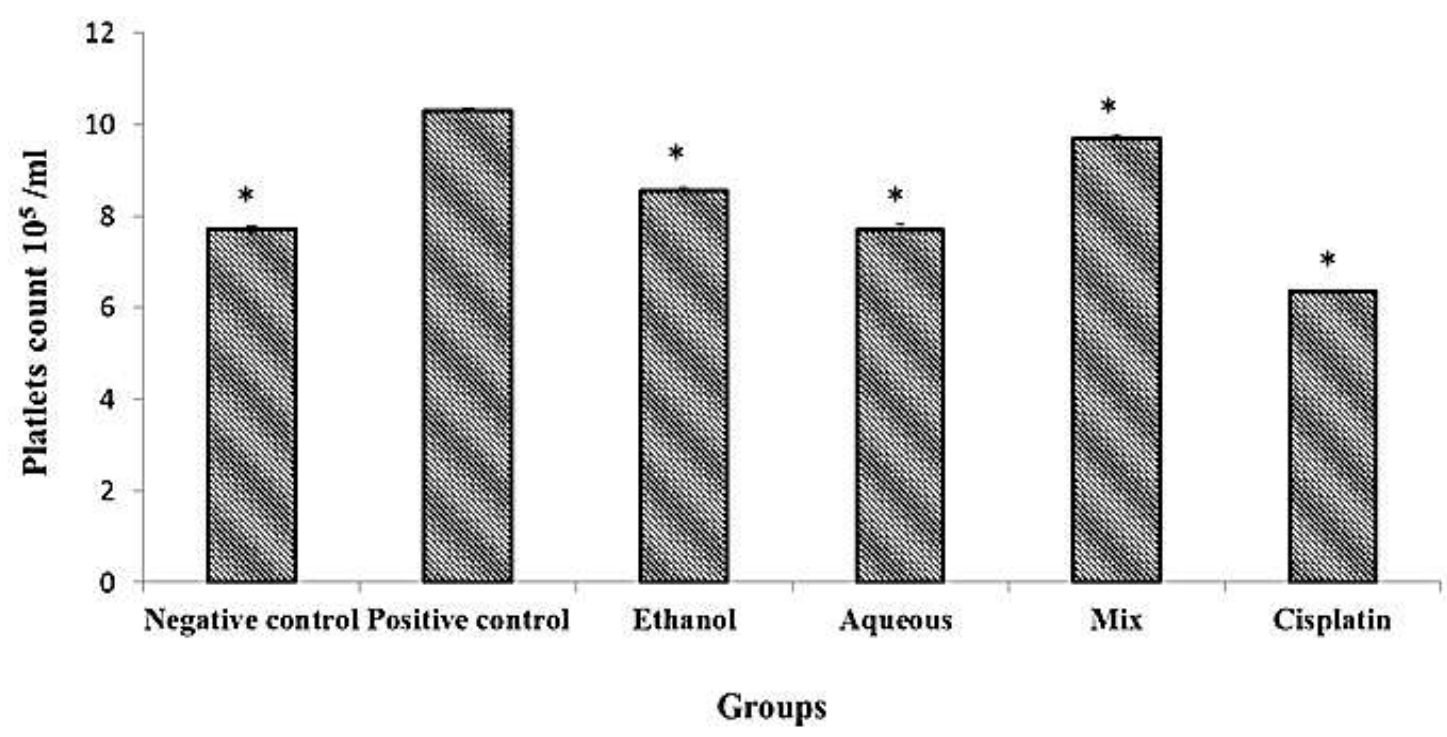

Figure 5. Changes in platelets count of EAC-bearing mice after 14 days of treatment with TW and cisplatin. The results are expressed as Mean \pm S.E.M. $(n=6)$. Statistically significant differences between experimental groups compared with EAC positive control group are denoted by $*(\mathrm{P} \leq 0.05)$.

\section{Effects of TW extracts on NLR, MLR and PLR.}

A significant decline has been reported in NLR, MLR and PLR ratios in response to treatment with both ethanolic and aqueous extracts of TW when compared with the EAC positive control group (Figs $6,7 \& 8$ ). Conversely, the cisplatin treated group showed nonsignificant changes in MLR ratio compared with the EAC positive control group, while PLR and NLR ratios revealed a significant decrease compared with the EAC positive control group. 


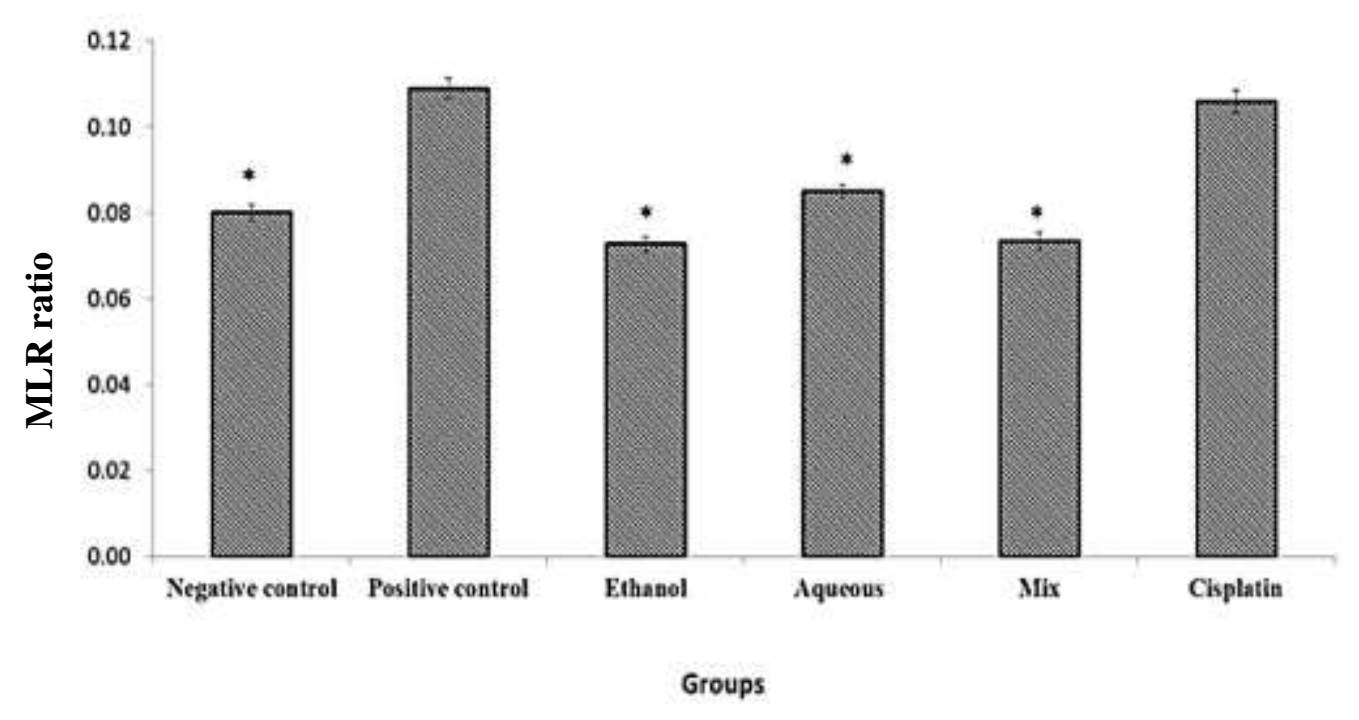

Figure 6. Changes in MLR ratio in the blood of EAC-bearing mice after 14 days of treatment with TW extracts and cisplatin. The results are expressed as Mean \pm S.E.M. $(n=$ 6). Statistically significant differences between experimental groups compared with EAC positive control group are denoted by $*(\mathrm{P} \leq 0.05)$.

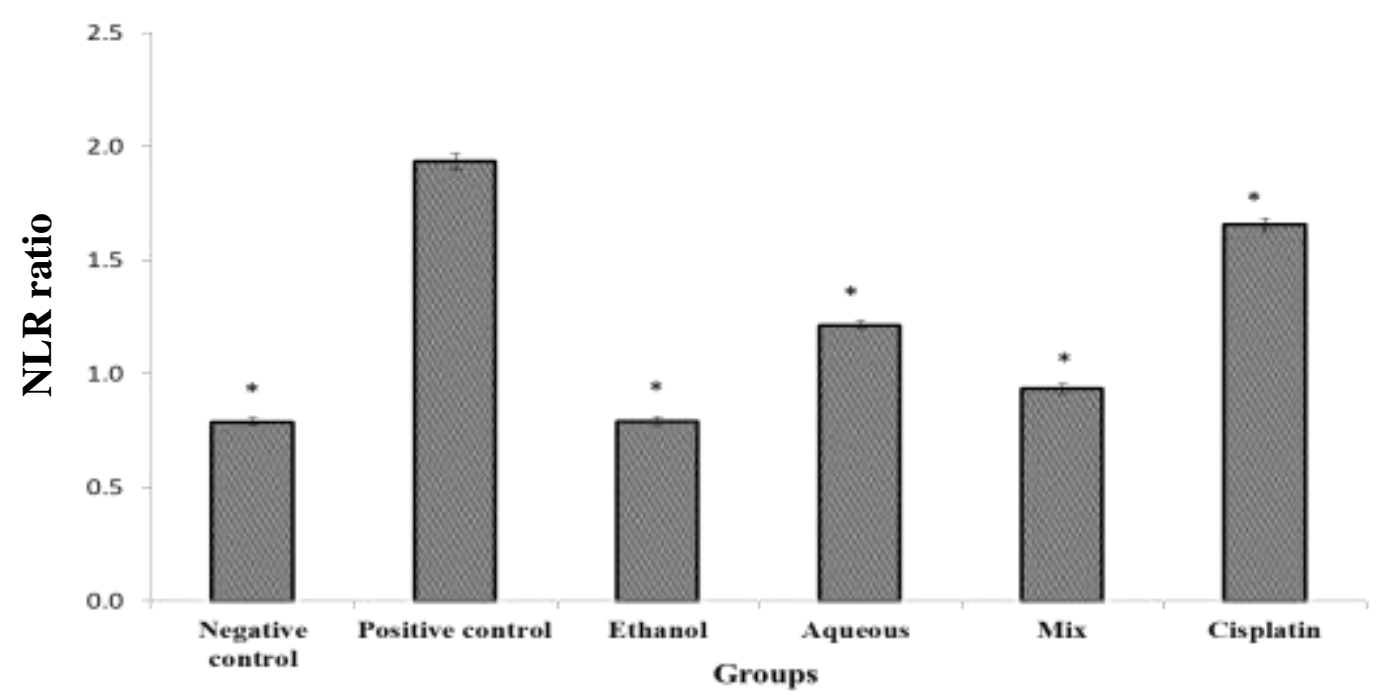

Figure 7. Changes in NLR ratio in the blood of EAC-bearing mice after 14 days of treatment with TW extracts and cisplatin. The results are expressed as mean \pm S.E.M. $(n=6)$. Statistically significant differences between groups compared with EAC positive control group are denoted by $*(\mathrm{P} \leq 0.05)$. 


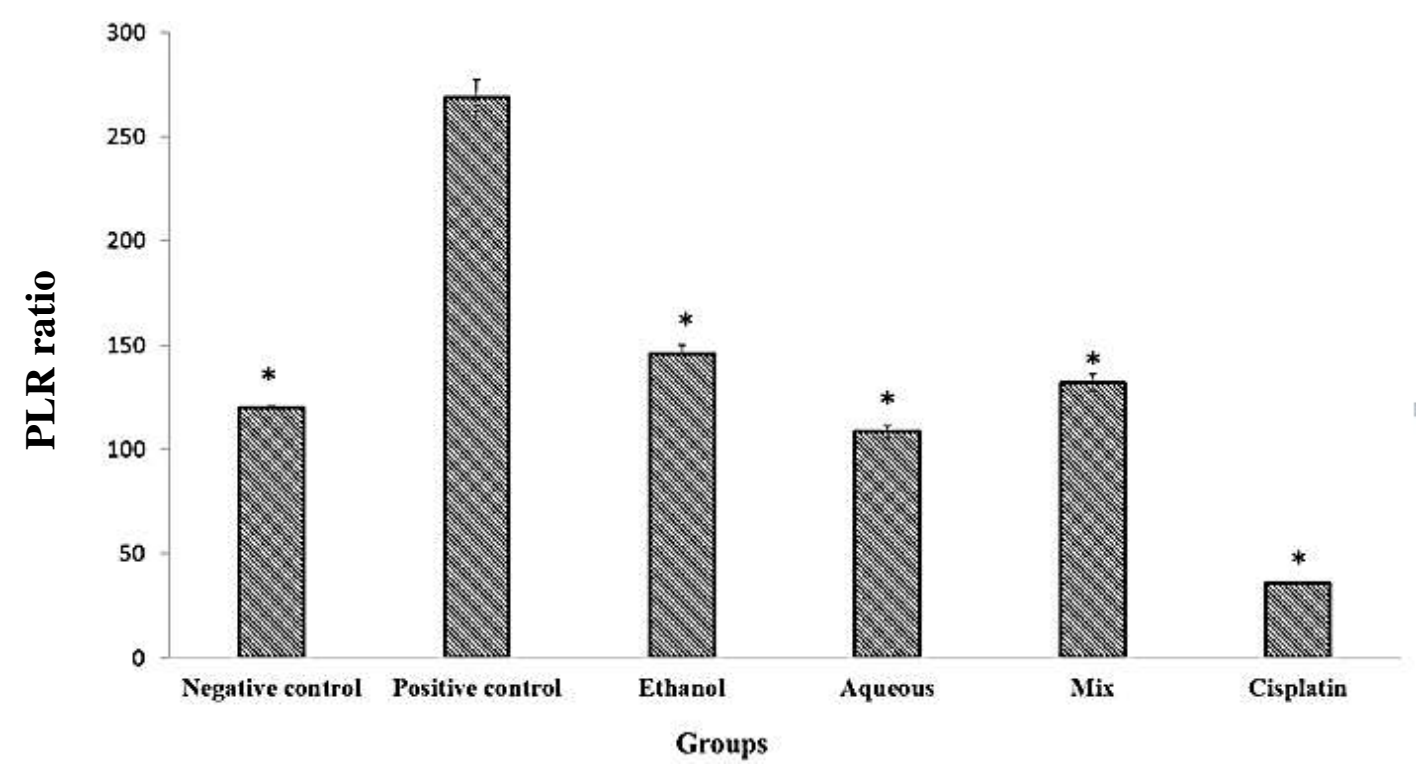

Figure 8. Changes in PLR ratio in the blood of EAC-bearing mice after 14 days of treatment with TW extracts and cisplatin. The results are expressed as Mean \pm S.E.M. $(n=6)$. Statistically significant differences between groups compared with EAC positive control group are denoted by $*(\mathrm{P} \leq 0.05)$.

\section{DISCUSSION}

The complete blood picture or count (CBC) is used as an extensive screening assessment to monitor many health problems such as anemia and infectious diseases, in addition to many other health disorders like cancer and immunodeficiencies [17]. CBC includes a group of measurements that scan different compartments of the blood, which play a significant role in metabolism and are an essential index for healthiness in both humans and animals [18]. It has been recently established that some values of CBC like NLR, MLR and PLR are associated with the activity, illness and mortality of various inflammatory diseases and cancer in particular [19]. Obviously, hematological parameters possess numerous advantages owing to the fact that they are reasonable and valid, so they can aid oncologists in growing countries in forecasting cancer progression [20].

Anemia and myelosuppression are the most common hematological conditions in cancer patients, and their prevalence rises with routine treatments such as chemotherapy/radiotherapy, which has a damaging effect on the bone marrow. For instance, RBCs count, HB level may be decreased through destruction and/or the inability of the bone marrow to produce these cells [21].

In the current study, a decline in RBC count, HB level, and PCV values were detected in tumor bearing mice. This decline could be due to iron insufficiency or hemolytic and myelopathic illnesses reported in cancer [22]. Recovery of the HB content, PCV, and 
ameliorating RBC count, almost near the normal values, was prominent in the treated mice with TW extracts indicating the protective action of the TW extract treatment on the hemopoietic system [23]. Most of the flavonoids, existing in the TW extracts, defend against the deformability of RBCs and also enhance their osmotic fragility, so the RBCs and their indices were ameliorated after treatment with the extracts [24].

The present work revealed a significant decrease in RBC count and related indices following treatment with cisplatin. Routinely used anticancer chemotherapies such as cisplatin has been reported to induce anemia, attack of normal tissue causing blood loss, bone marrow malfunction with erythropoiesis disruption, and efficient iron deficiency as a result of inflammation [25]. While, the secondary erythropoietin deficiency following cisplatin treatment is attributed to the induced renal tubular damage. Interestingly, treatment with TW extracts restored and maintained normal values of RBC counts, revealing their hematopoietic protective effect without inspiring the remarked myelotoxicity of classical anti-cancer chemotherapies [25].

Spontaneous neutrophilic leucocytosis is recognized in carcinogenesis, while substances with anticancer activity tend to recover this disorder. So, one of the trustworthy features for assessing an anticancer medication is a reduction in the blood total WBC count of the tumorized animals according to their antineoplastic action [26]. In the current work, induction of cancer in EAC mice showed a significant increase in the total number of WBC by almost two times compared to normal mice, where this finding was in line with previous studies [27]. Generation of myelotoxicity in EAC mice reflects the immunological importance of encountering the adversarial condition established by the chemotaxis of foreign bodies in the blood. Alternatively, an inflammatory reaction includes the infiltration of leukocytes, like neutrophils and monocytes, which are attracted by inflammatory mediators such as cytokines and chemokines that are released from cancer cells into the tumor microenvironment [28]. This reaction leads to redisposition of the vascular WBCs, which results in decreased lymphocyte number and increased neutrophil number. So, general inflammation can be demonstrated as neutrophilia, thrombocytosis, and relative lymphocytopenia [29].

Interestingly, our findings showed a noticeable decrease in WBC count and a rearranged distribution of neutrophils (\%) and lymphocytes (\%) in addition to platelet counts, but almost not to the normal values in all TW extracts treated mice. These results obviously elucidate their noteworthy antitumor effect against EAC. The antioxidants existing in the extracts could be accountable for this activity [23]. Likewise, phytochemical studies revealed the presence of many components such as flavonoids, alkaloids, and terpenoids in alcoholic extracts and they have been reported to possess anti-malignant and anti-mutagenic effects [30]. Leukopenia and lymphocytopenia have been reported after chemical treatment and remain one of the most common undesired effects of chemotherapy [25]. The current study revealed that treatment with cisplatin has a lymphopenic effect and induces an increase in neutrophils. It has been established that inflammatory reactions play a significant role in the growth and development, angiogenesis, and metastasis of many types of cancers [31]. A chemotherapeutic medication disrupts the adaptable immune reactions and modifies responses, leading to extraordinary inflammatory responses. Lymphocytes have an essential role in immune reactions as they detect and destroy malignant cells [32]. Furthermore, 
neutrophilia has a role in cancer propagation and metastasis involving the liberation of reactive oxygen species (ROS) and/or nitric oxide and restoration of the tumor extracellular matrix.

TW root extracts inhibit macrophage and lymphocyte release of pro-inflammatory cytokines and mediators, adhesion molecules, and matrix metalloproteinases [33]. So, the observed significant increase in lymphocytes in the TW extract treated group may indicate its immune modulating effect.

Extraction is the key procedure by which bioactive composites can be gotten from biomass of plant materials. Accordingly, the main goal of the extraction method is to maximize the quantity of the target composites and get the highest biological actions of these extracts, which are not affected only by the extraction procedure but also by the solvent used during the process [34]. Current data showed a noticeably superior effect of the ethanolic extract, the superiority of the alcoholic extract over that of the aqueous one could be due to the existence of phenolic and flavonoid composites, which are absent in the aqueous extract solution. Hence, alcoholic extract has higher antioxidant protective activity.

In the present work, an increased number of platelets counted was detected in the untreated EAC-bearing mice group. Notably, the platelet count was significantly decreased in the EAC-bearing mice after treatment with ethanolic and aqueous extracts in addition to cisplatin, demonstrating their obvious antiplatelet action. Increased blood platelet count is associated with survival and diagnosis in a variety of cancers, whereas promising antitumor agents should reduce the circulating platelet count [35]. Platelet count reduction inhibited and delayed tumor growth and improved patient survival in a variety of clinical and experimental models [36].

Parameters like red cell distribution width (RDW), neutrophils, lymphocytes, monocytes, platelets, and their ratios (NLR), (MLR) and (PLR) have been suggested as prospective diagnostic factors for cancer in a lot of studies [37].

Increased NLR and MLR denote relatively superior neutrophils or monocytes, and inferior lymphocyte counts. High NLR and MLR values indicate an unjustified immune reaction, which diminishes the predictability of anti-tumor resolution. As systemic inflammation leads to changes in the circulatory blood leukocytes, it is suggested that NLR and MLR values can be considered as an indicator for forecasting malignancy invasion and severity [38]. The results of the current study revealed a remarkable, significant decrease in NLR, MLR, and PLR values in the TW extract treated mice. The bioactive components derived from the traditional Chinese medicinal plants of the Celastraceae family, such as celastrol and triterpenoid, which are isolated from TW, are known to have anti-inflammatory and anti-oxidant properties, enabling their use in numerous pharmacological applications. The increased platelet count is mostly associated with an increase in tumor cell migration level where it provides the necessary growth factors needed whereas tumor cells reach their target organ. Consequently, the increased PLR ratio can be measured as a sign of tumor evolution and vascular invasion recognized in cancer [39]. A significant correlation between neutrophil count and platelet count in cancer progression has been reported, and lymphocyte count may be associated with tumor stage due to its intrinsic cellular nature immune response in cancer eradication [40]. 


\section{CONCLUSION}

The current study revealed a marked improvement in hematological parameters after treatment with TW extracts where cisplatin showed different side effects, such as anemia, leukopenia, and neutrophilia. Likewise, estimation of the NLR, MLR and PLR ratios, forecasting parameters as a prospective way of the invasiveness of cancer, was ameliorated after TW extracted treatment rather than cisplatin. Therefore, the usage of these values in the expectation of cancer invasiveness warrants further inclusive and forthcoming research studies before more employment in clinical applications to authorize these results and to clarify the prospective mechanism of common inflammatory reactions against cancer cells.

\section{REFERENCES}

[1] Bryer E, Henry D. International Journal of Clinical Transfusion Medicine. 2018;6:21-31. https://doi.org/10.2147/IJCTM.S187569

[2] Pravettoni G, Mazzocco K, Gorini A, and Curigliano G. Critical Reviews inOncology/Hematology,2016Apr;100:6973.doi:10.1016/j.critrevonc.2016.01.023

[3] Maina, R.N., D. Walsh, C. Gaddy, G. Hongo, J. Waitumbi, et al., Malaria Journal 9, S4 (2010). https://doi.org/10.1186/1475-2875-9-S3-S4

[4] Miller KD, Siegel RL, Lin CC, Mariotto AB, Kramer JL, et al., CA Cancer J Clin. 2016 Jul;66(4):271-89. doi: 10.3322/caac.21349

[5] Li, K., W.-c. Duan, H.-b. Zhao, L. Wang, W.-w. Wang, et al., Scientific Reports 9, 13239 (2019). https://doi.org/10.1038/s41598-019-49733-6

[6] Li, K., W.-c. Duan, H.-b. Zhao, L. Wang, W.-w. Wang, et al., The American Journal of Chinese Medicine. 2010;38(4):801-14. doi:10.1142/S0192415X10008251

[7] Chen YZ, Gao Q, Zhao XZ, Chen XM, Zhang F, et al., Mei CL. Internal Medicine. 2010;49(19):2049-55. doi:10.2169/internalmedicine.49.3704.

[8] Choi BS, Sapkota K, Kim S, Lee HJ, Choi HS, et al., SH-SY5Y. Neurochem Res. 2010 Aug;35(8):1269-80. doi:10.1007/s11064-010-0185-4.

[9] Wan, Y.-G., X.-Y. Che, W. Sun, Y.-R. Huang, X.-J. Meng, et al., Journal of

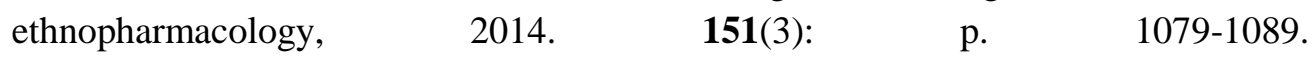
https://doi.org/10.1016/j.jep.2013.12.005.

[10] Chen SR, Dai Y, Zhao J, Lin L, Wang Y, Wang Y. Front Pharmacol. 2018 Feb 14;9:104. doi: 10.3389/fphar.2018.00104.

[11] He, M. F., Liu, L., Ge, W., Shaw, P. C., Jiang, R., Wu, L. W., et al., Journal of Ethnopharmacology, 2009. 121(1), p.6168. https://doi.org/10.1016/j.jep.2008.09.033

[12] Chan WY, Ng TB. Contraception. 1995 Jan;51(1):65-71. doi: 10.1016/00107824(94)00001-d.

[13] Abdallah NM, Noaman E, Eltahawy NA, Badawi AM, Kandil E, Mansour NA, Mohamed HE. Asian Pac J Cancer Prev. 2016; 17(9):43674375. doi:10.14456/apjcp.2016.267.

[14] Lipsky PE, Brinker AM, Ma J, Raskin I. Phytochemistry. 2007 Mar;68(6):732-66. doi: 10.1016/j.phytochem.2006.11.029.

[15] Sedlar, A., T. Dolinsek, B. Markelc, L. Prosen, S. Kranjc, et al.,. Radiol Oncol. 2012;46(4):302-311. doi:10.2478/v10019-012-0044-9. 
[16] Grant, Derek. "Schalm's veterinary hematology: 4th Ed, 1986. Food and Chemical Toxicology 25. 198;p. 628-629. doi:10.1016/0278-6915(87)90029-9.

[17] Omuse, G., D. Maina, J. Mwangi, C. Wambua, K. Radia, et al., PLoS One. 2018 Jun 7;13(6):e0198444. doi: 10.1371/journal.pone.0198444.

[18] Erian, N., H. Hamed, A. El-Khateeb, and M. Farid. Journal of Agricultural Chemistry and Biotechnology, 2015. 6(11): p. 559-578. doi: 10.21608/jacb.2015.48462

[19] Velidedeoglu M, Kundaktepe BP, Aksan H, Uzun H. Medicina (Kaunas). 2021;57(7):698. Published 2021 Jul 8. doi:10.3390/medicina57070698j

[20] Wiranata, S., I.A.W. Anjani, I.P.G.S. Saputra, I.G.A.S. Sadvika, I.P.Y. Prabawa, et al., Open Access Macedonian Journal of Medical Sciences. 8, B (Oct. 2020), 10581063. DOI:https://doi.org/10.3889/oamjms.2020.5336.

[21] Madeddu, C., G. Gramignano, G. Astara, R. Demontis, E. Sanna, et al., Frontiers in physiology, 2018. 9: p. 1294. doi:10.3389/fphys.2018.01294

[22] Raju Asirvatham, A.J.M.C., Journal of Complementary Medicine Research, 2013. 2(1): p. 9-14. doi:10.5455/jice.20120615104543.

[23] Liu, Z.; Ma, L.; Zhou, G.-B. Molecules 2011, 16, 5283-5297. https://doi.org/10.3390/molecules16065283.

[24] Igbokwe, N., Sokoto Journal of Veterinary Sciences, 2018. 16(4): p. 1-23. doi: 10.4314/sokjvs.v16i4.1.

[25] Bryer E, Henry D. International Journal of Clinical Transfusion Medicine. 2018;6:21-31. https://doi.org/10.2147/IJCTM.S187569.

[26] Rajkapoor, B., M. Sankari, M. Sumithra, J. Anbu, N. Harikrishnan, et al., Bioscience, biotechnology, and biochemistry, 2007. 71(9): p. 2177-2183. doi:10.1271/bbb.70149.

[27] Pai, K., P. Srilatha, K. Suryakant, M.M. Setty, P.G. Nayak, et al., Pharmaceutical biology, 2012. 50(3): p. 270-277. doi: 10.3109/13880209.2011.599035.

[28] Pereira JFS, Jordan P, Matos P. Immuno. 2021; 1(2):91-118. https://doi.org/10.3390/immuno1020007.

[29] uthrie GJ, Charles KA, Roxburgh CS, Horgan PG, McMillan DC, et al., Crit Rev Oncol Hematol. 2013 Oct;88(1):218-30. doi: 10.1016/j.critrevonc.2013.03.010.

[30] P. K. M. Nagarathna, K.Y., D. M. M. Lou and G. T. Bhutia, IJPSR (2019), Volume 10, P. 4868-4880 doi: 10.13040/IJPSR.0975-8232.10(11).4868-80

[31] Elegbede, A.A., A.J. Gibson, H. Fu, M.L. Dean, D.A. Ezeife, et al., American journal of clinical oncology, 2020. 43(4): p. 236-242. doi: https://doi.org/10.1097/COC.0000000000000657

[32] Gonzalez H, Hagerling C, Werb Z.. Genes Dev. 2018 Oct 1;32(19-20):1267-1284. doi: 10.1101/gad.314617.118.

[33] Kimura K, Nomi N, Yan ZH, Orita T, Nishida T. Mol Vis. 2011 Feb 18;17:526-32. PMID: 21364906; PMCID: PMC3044697.

[34] Truong, D.-H., D.H. Nguyen, N.T.A. Ta, A.V. Bui, T.H. Do, et al., Journal of food quality. 2019. https://doi.org/10.1155/2019/8178294

[35] Buergy, D., F. Wenz, C. Groden, and M.A. Brockmann, International journal of cancer, 2012. 130(12): p. 2747-2760. doi: 10.1002/ijc.27441.

[36] Cho, M.S., J. Li, R. Gonzalez-Delgado, H. Lee, M. Vasquez, et al., Blood Advances, 2021. 5(7): p. 1947-1951. doi: 10.1182/bloodadvances.2020003410 
[37] Gao, Y., W. Guo, S. Cai, F. Zhang, F. Shao, et al., Journal of Cancer, 2019. 10(14): p. 3188. doi: 10.7150/jca.30281

[38] Eid, S., H. Hasan, D. Abdel-Aleem, and A. Rayan, Journal of Cancer Therapy, 2019. 10(9): p. 755-777. doi:10.4236/jct.2019.109064

[39] Faria, S.S., P.C. Fernandes Jr, M.J.B. Silva, V.C. Lima, W. Fontes, et al., ecancermedicalscience, 2016. 10. doi: 10.3332/ecancer.2016.702

[40] Lecot Pacôme, Sarabi Matthieu, Pereira Abrantes Manuela, Mussard Julie, et al., Front. Immunol., (10) 2155 (2019)doi.org/10.3389/fimmu.2019.02155. 